\title{
A local energy market for electricity and hydrogen
}

\author{
Xiao, Yunpeng; Wang, Xifan; Pinson, Pierre; Wang, Xiuli
}

Published in:

IEEE Transactions on Power Systems

Link to article, DOI:

10.1109/TPWRS.2017.2779540

Publication date:

2018

Document Version

Peer reviewed version

Link back to DTU Orbit

Citation (APA):

Xiao, Y., Wang, X., Pinson, P., \& Wang, X. (2018). A local energy market for electricity and hydrogen. IEEE Transactions on Power Systems, 334, 3898-3908. https://doi.org/10.1109/TPWRS.2017.2779540

\section{General rights}

Copyright and moral rights for the publications made accessible in the public portal are retained by the authors and/or other copyright owners and it is a condition of accessing publications that users recognise and abide by the legal requirements associated with these rights.

- Users may download and print one copy of any publication from the public portal for the purpose of private study or research.

- You may not further distribute the material or use it for any profit-making activity or commercial gain

- You may freely distribute the URL identifying the publication in the public portal

If you believe that this document breaches copyright please contact us providing details, and we will remove access to the work immediately and investigate your claim. 


\title{
A Local Energy Market for Electricity and Hydrogen
}

\author{
Yunpeng Xiao, Student Member, IEEE, Xifan Wang, Fellow, IEEE, Pierre Pinson, Senior Member, IEEE, \\ and Xiuli Wang, Senior Member, IEEE
}

\begin{abstract}
The proliferation of distributed energy resources entails efficient market mechanisms in distribution-level networks. This paper establishes a local energy market (LEM) framework in which electricity and hydrogen are traded. Players in the LEM consist of renewable distributed generators (DGs), loads, hydrogen vehicles (HVs), and a hydrogen storage system (HSS) operated by a HSS agent (HSSA). An iterative LEM clearing method is proposed based on the merit order principle. Players submit offers/bids with consideration of their own preferences and profiles according to the utility functions. The decentralized LEM clearing process not only avoids complex calculation induced by centralized decision process, but also preserves players' privacy. Case studies are conducted that demonstrate that the LEM promotes local integration of renewable energy, reduces peak demand, and improves players' utilities. Sensitivity analysis is then implemented to discuss the influences on the LEM clearing results of capacities of DGs, Loads, and the HSS, as well as price of hydrogen from the hydrogen station (HS).
\end{abstract}

Index Terms-Distributed energy resources, hydrogen storage system, iterative market clearing, local energy market.

\section{NOMENCLATURE}

Indices

$i, j, k \quad$ Index of renewable DGs/Loads/HVs.

$t, l \quad$ Index of time slots.

$m_{j} \quad$ Index of non-interruptible and deferrable tasks of Loads.

$n_{j} \quad$ Index of interruptible and deferrable tasks of Loads.

$s \quad$ Index of scenarios.

$r \quad$ Index of iteration.

\section{Parameters}

$\begin{array}{ll}D^{H V} & \text { Travel distance of HV. (km) } \\ E^{\text {Loadid }} & \text { Overall electricity consumed by interruptible } \\ & \text { and deferrable tasks of Load }(\mathrm{kWh}) . \\ M o l^{H} & \text { Molar mass of hydrogen. }\left(\mathrm{kg} \cdot \mathrm{mol}^{-1}\right) \\ P^{A E L, \max } & \text { Maximum consumed electricity by electrolyz- } \\ & \text { er in an HSSA (kWh). } \\ P^{A E L, m i n} & \text { Minimum consumed electricity by electrolyzer } \\ & \text { in an HSSA (kWh). } \\ P^{A F C, \max } & \text { Maximum generated electricity by fuel cell of } \\ & \text { HSSA (kWh). } \\ P^{A F C, \min } & \text { Minimum generated electricity by fuel cell of } \\ & \text { HSSA (kWh). } \\ P^{D G} & \text { Electricity generated by DG (kWh). }\end{array}$

$P^{\text {Loadid,max }} \quad$ Maximum value of electricity consumed by interruptible and deferrable tasks of Load $(\mathrm{kWh})$.

$R \quad$ Gas constant. $\left(J \cdot \mathrm{mol}^{-1} \cdot K^{-1}\right)$

$T^{A}, T^{H} \quad$ Mean temperature inside hydrogen tank of HSSA/HV $(K)$

$V^{c a p}, V^{c a p A} \quad$ Capacity of hydrogen tank of HV/HSSA. $\left(m^{3}\right)$

$\lambda^{D P}, \lambda^{D S} \quad$ Purchasing/selling price of DisCo $(€ / \mathrm{kWh})$.

$\lambda^{H S} \quad$ Hydrogen price from hydrogen station $(€ / \mathrm{kg})$.

$t^{i d 1}, t^{i d 2} \quad$ Starting/ending time of interruptible and deferrable tasks of Load.

$t^{n i d 1}, t^{n i d 2}$

$t^{H V 1}, t^{H V 2}$

$\eta^{H V t r a}$

$\eta^{A E L}, \eta^{A F C}$

$\rho^{A, i n i}$

$\rho^{A, \max }$

$\rho^{A, \min }$

$\rho^{H V, i n i}$

$\rho^{H V, \max }$

$\rho^{H V, \text { min }}$

$\mu^{H}$

$\sigma_{s}$

$\varepsilon^{D G}, \varepsilon^{\text {Load }}$

$\triangle t$

Earliest/latest starting time of non-interruptible and deferrable tasks of Load.

Departure/arrival time of HV.

Efficiency of hydrogen over distance of $\mathrm{HV}$. $(\mathrm{kg} / \mathrm{km})$

Efficiency of electrolyzer/fuel cell of HSSA.

Initial pressure of hydrogen tank of HSSA (Pa).

Maximum pressure of hydrogen tank of HSSA (Pa).

Minimum pressure of hydrogen tank of HSSA (Pa).

Initial pressure of hydrogen tank of $\mathrm{HV}(\mathrm{Pa})$.

Maximum pressure of hydrogen tank of HV (Pa).

Minimum pressure of hydrogen tank of $\mathrm{HV}(\mathrm{Pa})$. Price difference between $\lambda^{H S}$ and $\lambda^{H L}(€ / \mathrm{kg})$.

Weight of scenario $s$.

Adjustment of offering/bidding prices for DG/Load.

Time resolution $(=1 \mathrm{~h})$.

Variables

$P^{A D P}$
$P^{A D S}$
$P^{A L P}$
$P^{A L S}$
$P^{A b i d}$
$P^{A o f f e r}$
$P^{D G D}$
$P^{D G L}$
$P^{D G o f f e r}$
$P^{\text {DGdeal }}$
$P^{\text {Load }}$
$P^{\text {LoadD }}$
$P^{\text {LoadL }}$

Electricity purchased from DisCo by HSSA (kWh). Electricity sold to DisCo by HSSA (kWh). Electricity purchased in LEM by HSSA $(\mathrm{kWh})$. Electricity sold in LEM by HSSA (kWh).

Bidding amount of HSSA (kWh).

Offering amount of HSSA (kWh).

Electricity sold to DisCo by DG (kWh).

Electricity sold in LEM by DG (kWh).

Offering amount of DG (kWh).

Accepted amount of DG ( $\mathrm{kWh})$.

Electricity consumed by Load (kWh).

Electricity purchased from DisCo by Load (kWh).

Electricity purchased in LEM by Load (kWh). 


\begin{tabular}{|c|c|}
\hline$P^{\text {Loadnind }}$ & $\begin{array}{l}\text { Electricity consumed by non-interruptibl } \\
\text { and non-deferrable tasks of Load (kWh). }\end{array}$ \\
\hline Loadid & $\begin{array}{l}\text { lectricity consumed by interruptible and } \\
\text { leferrable tasks of Load }(\mathrm{kWh}) \text {. }\end{array}$ \\
\hline$P^{\text {Loadnid }}$ & $\begin{array}{l}\text { lectricity consumed by non-interruptible } \\
\text { nd deferrable tasks of Load }(\mathrm{kWh}) \text {. }\end{array}$ \\
\hline$P^{\text {Loadbid }}$ & Bidding amount of Load (kWh). \\
\hline$P^{\text {Loaddeal }}$ & Accepted amount of Load (kWh). \\
\hline$Q^{H A L}$ & Hydrogen sold in LEM of HSSA (kg). \\
\hline$Q^{H A S}$ & $\begin{array}{l}\text { Hydrogen purchased from hydrogen station } \\
\text { of HSSA }(\mathrm{kg}) \text {. }\end{array}$ \\
\hline$Q^{H V L}$ & Hydrogen purchased in LEM of HV $(\mathrm{kg})$. \\
\hline$Q^{H V S}$ & $\begin{array}{l}\text { Hydrogen purchased from hydrogen station } \\
\text { of } \mathrm{HV}(\mathrm{kg}) \text {. }\end{array}$ \\
\hline$Q^{H V P}$ & Total purchased hydrogen of $\mathrm{HV}(\mathrm{kg})$. \\
\hline$Q^{H V U}$ & Total consumed hydrogen of HV (kg). \\
\hline$Q^{H V b i d}$ & Bidding amount of hydrogen of HV $(\mathrm{kg})$. \\
\hline$\lambda^{A b i d}$ & Bidding price of HSSA $(€ / \mathrm{kWh})$. \\
\hline$\lambda^{\text {Aoffer }}$ & Offering price of HSSA ( $€ / \mathrm{kWh})$. \\
\hline$\lambda^{\operatorname{Ln} A}$ & $\begin{array}{l}\text { Electricity price in LEM without HSSA } \\
(€ / \mathrm{kWh}) \text {. }\end{array}$ \\
\hline$\lambda^{L}$ & Electricity price in LEM ( $€ / \mathrm{kWh})$. \\
\hline$\lambda^{H L}$ & Hydrogen price in LEM ( $€ / \mathrm{kg})$. \\
\hline$\lambda^{D G o f f e r}$ & Offering price of DG $(€ / \mathrm{kWh})$. \\
\hline$\lambda^{\text {Loadbid }}$ & Bidding price of Load ( $€ / \mathrm{kWh})$. \\
\hline$\lambda^{H V b i d}$ & Bidding price of $\mathrm{HV}(€ / \mathrm{kg})$. \\
\hline$\rho^{H V}, \rho^{A}$ & $\begin{array}{l}\text { Pressure of hydrogen tank of } \mathrm{HV} / \mathrm{HSSA} \\
(\mathrm{Pa}) \text {. }\end{array}$ \\
\hline$u^{A P}, u^{A S}$ & $\begin{array}{l}\text { Binaries indicating operation mode of } \\
\text { HSSA. }\end{array}$ \\
\hline$u^{\text {nid }}$ & $\begin{array}{l}\text { Binary indicating operation mode of non- } \\
\text { interruptible and deferrable tasks of Load. }\end{array}$ \\
\hline$C^{A}$ & Cost of HSSA (€). \\
\hline$C^{H V}$ & Cost of HV (€). \\
\hline$C^{\text {Load }}$ & Cost of Load $(€)$. \\
\hline$U^{D G}$ & Utility of DG (€). \\
\hline$U^{L M}, U^{L}$ & Social welfare of LEM without/with \\
\hline
\end{tabular}
$(€)$.

\section{INTRODUCTION}

D ISTRIBUTED energy resources (DERs) including DGs, flexible loads and energy storage systems (ESSs) have been observed to proliferate and eventually take up a remarkable share in distribution-level networks [1]-[4]. In order to efficiently adopt DERs and properly evaluate their potential impacts, importance should be attached to the trading framework of DERs [5].

On the one hand, some studies focus on proposing trading strategies for DERs under current market mechanisms, which can be categorized as two groups, according to whether an energy aggregator (EA) exists. The EA, often served by the DisCo, can manage a microgrid or a virtual power plant [2], [6]-[8], and maximize the overall utility. Some of the EAs may possess DGs or ESSs to hedge against risks [9]. However, this attribute results in problems regarding allocation of profits/costs among players, as well as computational difficulties introduced by the increasing number of players.
Moreover, players' privacy is sacrificed. In the absence of EAs, consensus-based and ADMM (alternating direction method of multipliers) -based distributed scheduling methods are proposed [10]-[12]. These methods are dependent on the in-time information exchanges. Transactive energy is also emerging for coordinated operation of vast numbers of actively involved DERs based on value-based information in a smart grid [13].

On the other hand, the increasing penetration of DERs resulting in large-scale coexistence of generation and consumption in the vicinity of a neighborhood, together with the enhancement of communication and automatic control methods in a smart-grid environment, enable the creation of a LEM to trade DERs within the distribution level, also referred to as a decentralized or community energy market [1], [14][20]. For example, in the U.S., a green energy market has been established, only for trading renewable and uncertain energy: A large number of utilities, combining several kinds of energy sources and small-scale generating units, are now participating in this green energy market [1], [19]. In the Netherlands, a platform to trade customers' self-generated power has been implemented, in which the trade happens in peers under certain contracts [20]. Shamsi et al. [17] develop an economic dispatch problem considering mutual trade among agents within a local energy market in the structure of a community microgrid installed in the Missouri. Parag et al. [14] thoroughly discuss potential local trading manners for prosumers, such as decentralized, autonomous and flexible peer-to-peer models and prosumer community groups. Sikdar et al. [16] merge electric vehicles and electricity sources in a decentralized market based on random matching and subsequent bargaining. However, a complete local energy market can include many sources of uncertainty and flexibility, such as renewable DGs, flexible demands, and storage. Few studies ever concentrate on this situation, in which multiple sorts of independent participants exist. Furthermore, since the marginal production cost of renewable DGs is often deemed as zero, pricing of the zero-marginal-production-cost market is an indispensable issue when designing a LEM, and neither has this been discussed by current literature.

Since the ESS shows indispensable potentials in mitigating fluctuating generation, much more attention has been paid to trading strategies of ESS, which can be categorized in three groups. First, the ESS is combined with uncertain generations to reduce the revenue loss caused by forecast errors and to arbitrage in dynamic prices [22]. Second, the ESS is owned by a third party and the capacity is shared by players [23]. Third, the ESS participates in the electricity as an independent stakeholder to maximize its own utility [24]-[25]. As for the trading strategy of independent ESSs, most studies only consider the trade towards wholesale market. However, a distribution-level ESS can also trade directly to consumers for more profitability as other DERs. Few works focus on this two-sided trading.

Among the wide variety of types of ESS, the HSS has become a promising approach, since the whole electricityhydrogen conversion process is carbon free and only utilizes water. The HSS consists of an electrolyzer, a hydrogen storage tank and a fuel cell. Hydrogen is produced from 
electrolysis and stored in the hydrogen storage tank, and the fuel cell is used to generate electricity by hydrogen. Hydrogen can be purchased from a hydrogen station (HS) and sold to hydrogen consumers, eg. HVs [26]-[27]. The detailed operation principles of HSS are beyond the scope of this paper. The coordination of electricity and hydrogen has attracted increasing attention in current studies. The HSS can be utilized to improve revenues of wind generation [28] and for electricity retailers [29]. You et al. [30] further assess the value of an independent HSS in a multi-energy system containing electricity, heat, and gas.

The contributions of this work include the following. First, a LEM framework is established, in which both electricity and hydrogen are traded. Players of the LEM include renewable DGs, flexible and non-flexible loads, electric vehicles (EVs), HVs and an HSS. Since the LEM is organized at distribution level, players can choose to participate in the LEM or trade with DisCo/HS. To compare the advantages/disadvantages of trading in the LEM and with $\mathrm{DisCo} / \mathrm{Hs}$ and to address the influence of the HSS in the LEM, three cases are discussed in Section V. Then an iterative LEM clearing method is proposed based on the merit order principle. The whole LEM clearing procedure only requires players' offering or bidding parameters (prices and amounts) instead of their preferences and profiles (detailed utilization parameters of each appliance), thus preserving their privacy. As for the ESS, an HSS is adopted and operated by the HSSA. The proposed models and methods can be similarly applied where the ESS is in other forms.

For the sake of clarity, the following assumptions are made.

1. All players participating in the LEM are rational and have self-interest in their own objectives.

2. Network constraints are not taken into account, assuming all the players are connected to one feeder.

3. Strategic behaviors of the players are not taken into account, since players have no information or prior about the others' parameters during the iterative process.

The organization of the paper is as follows. Section II describes the system and problems in detail. Then Section III formulates mathematical models for LEM players. The iterative LEM clearing method is proposed in Section IV. Test cases and results analysis are conducted in Section V, and Section VI follows to conclude the paper.

\section{System ANd PRoblem Description}

This paper considers a LEM with 4 sorts of players, including renewable DGs, Loads (flexible, non-flexible, EVs), an HSSA, and HVs. Fig. 1 demonstrates an overall view of the LEM. The rectangle in dotted line represents the distribution network. Directions of power and hydrogen flows are demonstrated. Renewable DGs with zero marginal production costs sell electricity to the DisCo or the LEM. Loads are households in this paper, including flexible tasks, non-flexible tasks and EVs. Each household decides on portfolios in purchasing electricity from the DisCo and LEM based on its own utility function. The HSSA can either purchase or sell electricity with both the DisCo and the LEM. Furthermore, it can purchase

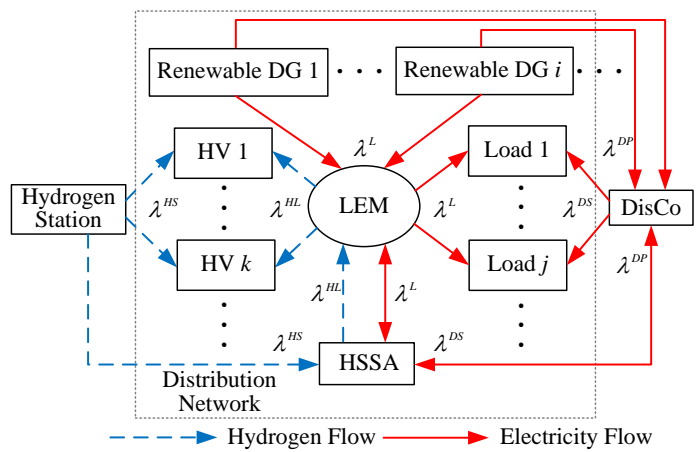

Fig. 1. Overall view of the LEM.

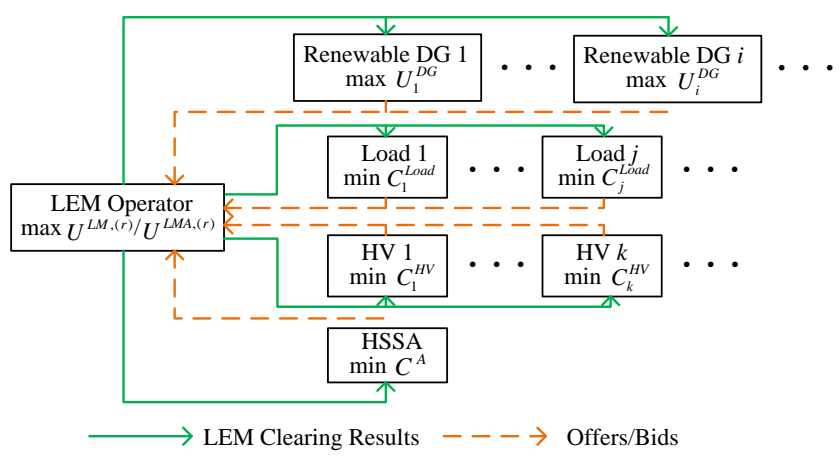

Fig. 2. Iterative LEM clearing method.

hydrogen from the HS and sell hydrogen to the LEM. HVs purchase hydrogen either from the HS or in the LEM. Note that the HS is assumed to acquire hydrogen directly from hydrogen-producing companies, instead of by electrolyzing water. Otherwise, the HS can be considered as the HSSA. The LEM operator is in charge of gathering offering/bidding parameters (prices and amounts) from different players aiming at maximizing social surplus. The LEM operator also broadcasts clearing results. The DisCo sells electricity to Loads and purchases electricity from renewable DGs and the HSSA, while the HS provides hydrogen to HVs and the HSSA.

Note that prices of selling and purchasing electricity with the DisCo are settled based on wholesale day-ahead prices, thus the LEM is actually a day-ahead market. The energy deviations from the scheduled quantities in the LEM are balanced with the DisCo at the selling/purchasing price $\left(\lambda^{D S} / \lambda^{D P}\right)$.

Since the LEM is set at distribution level, the number of players can be large and players differ in utility/cost features. To preserve privacy, players broadcast their offers/bids in the LEM instead of preferences and profiles (detailed utilization parameters of each appliance), and compete on both price and amount. Therefore, the competition among the players in this setting is neither Bertrand (competition on price) nor Nash-Cournot (competition on amount), but rather a mixture of both. Therefore, different from the problems where the players determine only the optimal amount or the optimal price for a particular amount, which can be solved by forming a Mathematical Program with Equilibrium Constraints [31]-[32], an intermediary step is anticipated to determine the offers/bids for players before the market-clearing process. Furthermore, with the help of smart-grid technologies, the LEM can be 
cleared in an iterative manner and no central entity is needed, indicating the players' privacy is preserved. Each player can make in-time decisions on its own offering/bidding strategies, and the market-clearing process is transparent with results broadcast promptly [8] [33]. On the one hand, thanks to advanced metering infrastructure and communication protocols, information regarding market prices and offering/bidding parameters is exchanged in the LEM promptly. On the other hand, each player is equipped with unit management modules [8] embedded in their appliances, which are responsible for autonomously controlling the player's behavior based on market clearing results as well as generating and submitting offering/bidding parameters to the market operator. As shown in Fig. 2, in the market-clearing process, each player submits its offering/bidding parameters to the market operator, who clears the LEM based on the merit order principle to maximize social welfare, and then broadcasts the results to all players. Each player then adapts its offering/bidding parameters based on the cleared results aiming at maximizing its own utility. The offering/bidding parameters will be sent back to the LEM operator and the LEM will be cleared again, until no adjustment on players' behavior is observed.

\section{Mathematical Models for Players}

This section illustrates mathematical models for players to maximize their utilities. The players can use these to generate their offering/bidding parameters, according to Fig. 2.

\section{A. Renewable Distributed Generator}

For renewable DG $i$, the objective function is

$$
\begin{array}{ll}
\max _{\Gamma_{i}^{D G}}^{D G} & U_{i}^{D G}=\sum_{s} \sigma_{s} \sum_{t}\left(\lambda_{t}^{D P} P_{i, t, s}^{D G D}+\lambda_{t}^{L} P_{i, t, s}^{D G L}\right) \\
\text { s.t. } & P_{i, t, s}^{D G D}+P_{i, t, s}^{D G L}=P_{i, t, s}^{D G}
\end{array}
$$

where $\Gamma_{i}^{D G}=\left\{P_{i, t, s}^{D G D}, P_{i, t, s}^{D G L} ; \forall i, t, s\right\}$ is the variable set. The objective of a renewable DG is to achieve the maximum value of overall profit in selling electricity to the DisCo and in the LEM, as represented in (1). The sum of electricity sold to the DisCo and in the LEM equals total generation, as (2).

\section{B. Load}

Load $j$ consists of three sorts of tasks, including interruptible and deferrable tasks, non-interruptible and deferrable tasks, as well as non-interruptible and non-deferrable tasks.
The objective function of Load $j$ is

$$
\min _{\Gamma_{j}^{L \text { oad }}} C_{j}^{\text {Load }}=\sum_{s} \sigma_{s} \sum_{t}\left(\lambda_{t}^{D S} P_{j, t, s}^{\text {LoadD }}+\lambda_{t}^{L} P_{j, t, s}^{\text {LoadL }}\right)
$$

s.t.

$$
\begin{aligned}
& P_{j, t, s}^{\text {LoadD }}+P_{j, t, s}^{\text {LoadL }}=P_{j, t, s}^{\text {Load }} \\
& P_{j, t, s}^{\text {Load }}=P_{j, t, s}^{\text {Loadnind }}+\sum_{n_{j}} P_{j, n_{j}, t, s}^{\text {Loadid }}+\sum_{m_{j}} P_{j, m_{j}, t, s}^{\text {Loadnid }} \\
& \sum_{t} P_{j, n_{j}, t, s}^{\text {Loadid }}=E_{j, n_{j}, s}^{\text {Loadid }} \\
& P_{j, n_{j}, t, s}^{\text {Loadid }}=0, \text { when } t \notin\left(t_{j, n_{j}, s}^{i d 1}, t_{j, n_{j}, s}^{i d 2^{\prime}}\right) \\
& 0 \leq P_{j, n_{j}, t, s}^{\text {Loadid }} \leq P_{j, n_{j}, s}^{\text {Loadid }, \text { max }} \\
& u_{j, m_{j}, t, s}^{\text {nid }} \in\{0,1\} \\
& u_{j, m_{j}, t, s}^{\text {nid }}=0, \text { when } t \notin\left(t_{j, m_{j}, s}^{\text {nid }}, t_{j, m_{j}, s}^{\text {nid }}\right) \\
& \sum_{t} u_{j, m_{j}, t, s}^{\text {nid }}=1 \\
& P_{j, m_{j}, t, s}^{\text {Loadnid }}=\sum_{l} P_{j, m_{j}, l, s}^{\text {Loadnid }} u_{j, m_{j}, t, s}^{\text {nid }}
\end{aligned}
$$

where $\Gamma_{j}^{L o a d}=\left\{P_{j, t, s}^{L o a d D}, P_{j, t, s}^{L o a d L} ; \forall j, t, s\right\}$ is the variable set. $P_{j, m_{j}, l, s}^{L \text { Ladid }}$ represents the predefined profiles of the task. The objective of a Load is to achieve the minimum value of overall cost in purchasing electricity from the DisCo and in the LEM, as represented in (3). The sum of electricity purchased from the DisCo and in the LEM equals the total consumption, and also equals the sum of electricity consumed by the three sorts of tasks, as shown in (4) and (5). Constraints (6)-(8) are for interruptible and deferrable tasks. The overall electricity consumption is constrained in (6). Equation (7) represents the starting and ending time, and the maximum electricity consumption of task $n_{j}$ is limited as (8) limits. Constraints (9)(12) are for non-interruptible and deferrable tasks. Equations (9)-(11) constrain the binary representing the starting time of task $m_{j}$. The optimized profile of task $m_{j}$ is determined by the predefined profile, as captured (12).

\section{Hydrogen Vehicle}

For the HV $k$, the objective function is

$$
\min _{\Gamma_{k}^{H V}} C_{k}^{H V}=\sum_{s} \sigma_{s} \sum_{t}\left(\lambda_{t}^{H S} Q_{k, t, s}^{H V S}+\lambda_{t}^{H L} Q_{k, t, s}^{H V L}\right)
$$

s.t.

$$
\begin{aligned}
& Q_{k, t, s}^{H V P}=Q_{k, t, s}^{H V S}+Q_{k, t, s}^{H V L} \\
& Q_{k, t, s}^{H V P}=0, \text { when } t \in\left(t_{k, s}^{H V 1}, t_{k, s}^{H V 2}\right) \\
& Q_{k, t, s}^{H V U}=0, \text { when } t \notin\left(t_{k, s}^{H V 1}, t_{k, s}^{H V 2}\right) \\
& D_{k, t, s}^{H V} \eta_{k}^{H V t r a}=Q_{k, t, s}^{H V U} \\
& \rho_{k, t, s}^{H V}=\rho_{k, t-1, s}^{H V}+\frac{R T_{k}^{H}}{V_{k}^{c a p} M o l^{H}}\left(Q_{k, t, s}^{H V P}-Q_{k, t, s}^{H V U}\right) \\
& \rho_{k}^{H V, m i n} \leq \rho_{k, t, s}^{H V} \leq \rho_{k}^{H V, m a x} \\
& \rho_{k, t_{0}, s}^{H V}=\rho_{k, t_{T}-1, s}^{H V}=\rho_{k}^{H V, i n i}
\end{aligned}
$$

where $\Gamma_{k}^{H V}=\left\{Q_{k, t, s}^{H V S}, Q_{k, t, s}^{H V L} ; \forall k, t, s\right\}$ is the variable set. The objective of an $\mathrm{HV}$ is to achieve the minimum value 
of overall cost in purchasing hydrogen from the HS and in the LEM, as represented in (13). The total purchased hydrogen equals the sum of that purchased from the HS and in the LEM, as shown in (14). The purchasing and utilization time of hydrogen of the HV are presented in (15) and (16). Equation (17) stands for the need for hydrogen to operate the HV. Constraints (18)-(20) are for the hydrogen tank of the $\mathrm{HV}$, where the hydrogen pressure is calculated by (18), the boundary of hydrogen pressure is ensured by (19), and the initial value is forced by (20).

\section{Hydrogen Storage System Agent}

For the HSSA, the objective function is

$$
\begin{gathered}
\min _{\Gamma^{A}} C^{A}=\sum_{t}\left(\lambda_{t}^{D S} P_{t}^{A D P}-\lambda_{t}^{D P} P_{t}^{A D S}+\lambda_{t}^{L} P_{t}^{A L P}\right. \\
\left.-\lambda_{t}^{L} P_{t}^{A L S}+\lambda_{t}^{H S} Q_{t}^{H A S}-\lambda_{t}^{H L} Q_{t}^{H A L}\right)
\end{gathered}
$$

s.t.

$$
\begin{aligned}
& Q_{t}^{H A L}=\sum_{k} Q_{k, t}^{H V L} \\
& \rho_{t}^{A}=\rho_{t-1}^{A}+\frac{R T^{A}}{V^{c a p A} M o l^{H}}\left(\frac{\eta^{A E L}\left(P_{t}^{A D P}+P_{t}^{A L P}\right)}{L H V^{H}} \Delta t M_{o l}^{H}\right. \\
& \left.\quad-\frac{P_{t}^{A D S}+P_{t}^{A L S}}{\eta^{A F C} L H V^{H}} \Delta t M o l^{H}+Q_{t}^{H A S}-Q_{t}^{H A L}\right) \\
& \rho^{A, m i n} \leq \rho_{t}^{A} \leq \rho^{A, \max } \\
& \rho_{t_{0}}^{A}=\rho_{t_{T}-1}^{A}=\rho^{A, i n i} \\
& P_{t}^{A D P}+P_{t}^{A L P} \leq u_{t}^{A P} P^{A E L, \max } \\
& P_{t}^{A D P}+P_{t}^{A L P} \geq u_{t}^{A P} P^{A E L, \min } \\
& P_{t}^{A D S}+P_{t}^{A L S} \leq u_{t}^{A S} P^{A F C, \text { max }} \\
& P_{t}^{A D S}+P_{t}^{A L S} \geq u_{t}^{A S} P^{A F C, \text { min }} \\
& u_{t}^{A P}, u_{t}^{A S} \in\{0,1\}, u_{t}^{A P}+u_{t}^{A S} \leq 1 \\
& \lambda_{t}^{H L}=\lambda_{t}^{H S}-\mu^{H}
\end{aligned}
$$

where $\Gamma^{A}=\left\{P_{t}^{A D P}, P_{t}^{A D S}, P_{t}^{A L P}, P_{t}^{A L S}, Q_{t}^{H A S}, Q_{t}^{H A L} ; \forall t\right\}$ is the variable set. The objective of the HSSA is to achieve the minimum value of overall cost in trading electricity and hydrogen with the DisCo, HS and LEM, as represented in (21). The amount of hydrogen traded in the LEM is constrained in (22). Constraints (23)-(25) are for the hydrogen tank of the HSSA, where the hydrogen pressure is calculated by (23), the boundary of the hydrogen pressure is ensured by (24), and the initial value is forced by (25). Constraints (26)-(30) provide the limits for hydrogen and electricity production by the HSSA. Note that hydrogen production and electricity production cannot be carried out at the same time. Equation (31) shows the relation between hydrogen price from the HS and in the LEM. According to (13)-(20), it is clear to see that when $\mu^{H}>0$ in (31), HVs will purchase hydrogen from the HSSA up to the maximum the HSSA can provide.

\section{The Iterative Local Energy Market Clearing METHOD}

\section{A. The Local Energy Market Clearing Principle}

As stated in Section II, the LEM clearing method is in an iterative manner. The players adjust their offering/bidding parameters after the LEM is cleared, and then the LEM is cleared again with adjusted parameters and broadcasts the results to players until no adjustment of players' offering/bidding parameters is observed. In each iteration process, the LEM clearing method should result in maximizing social welfare and it should respect the merit order principle. However, if the supply and demand curves fail to intersect, the LEM price will be settled as the price of the last unit of accepted amount on the curve that has a surplus [34]. Since the players are able to decide whether to participate in the LEM, their offering/bidding parameters should assure that they achieve higher utilities in the LEM than in trading with the DisCo/HS.

\section{B. Offering/Bidding Parameters}

Since players want to achieve higher utilities through every iteration, they determine their offering/bidding parameters based on the utility values in the last iteration. Specifically, the offering/bidding parameters are generated according to the utility/cost of trading with the DisCo in the first iteration.

For DG $i$, if the accepted amount of electricity in the LEM is $P_{i, t}^{D \text { Gdeal },(r-1)}$ at the price of $\lambda_{t}^{L,(r-1)}$ in the $r-1$ th iteration, then the offering parameters in the $t$ th iteration are

$\lambda_{i, t}^{D G o f f e r,(r)}=\left\{\begin{array}{l}\lambda_{t}^{D P}, \text { if } P_{i, t}^{D G o f f e r,(r)} \in S_{i, t}^{D G 1,(r)} \\ \lambda_{t}^{L,(r-1)}, \text { if } P_{i, t}^{D G o f f e r,(r)} \in S_{i, t}^{D G 2,(r)}\end{array}\right.$

where $S_{i, t}^{D G 1,(r)}=\left[0, \frac{1}{N_{s}} \sum_{s} P_{i, t, s}^{D G}-P_{i, t}^{D G d e a l,(r-1)}\right], S_{i, t}^{D G 2,(r)}=$ $\left[\frac{1}{N_{s}} \sum_{s} P_{i, t, s}^{D G}-P_{i, t}^{D \text { Gdeal },(r-1)}, \frac{1}{N_{s}} \sum_{s} P_{i, t, s}^{D G}\right]$. Note that $\lambda_{t}^{L,(r-1)}>$ $\lambda_{t}^{D P}$, otherwise the DG would not participate in the $r-1$ th iteration of LEM.

Similarly, for Load $j$, the bidding parameters are

$\lambda_{j, t}^{\text {Loadbid },(r)}=\left\{\begin{array}{l}\lambda_{t}^{D S}, \text { if } P_{j, t}^{\text {Loadbid },(r)} \in S_{j, t}^{\text {Load } 1,(r)} \\ \lambda_{t}^{L,(r-1)}, \text { if } P_{j, t}^{\text {Loadbid },(r)} \in S_{j, t}^{\text {Load } 2,(r)}\end{array}\right.$

where $S_{j, t}^{\text {Load } 1,(r)}=\left[0, \frac{1}{N_{s}} \sum_{s} P_{j, t, s}^{\text {Load },(r)}-P_{j, t}^{\text {Loaddeal },(r-1)}\right]$, $S_{j, t}^{\text {Load } 2,(r)}=\left[\frac{1}{N_{s}} \sum_{s} P_{j, t, s}^{\text {Load },(r)}-P_{j, t}^{\text {Loaddeal },(r-1)}, \frac{1}{N_{s}} \sum_{s} P_{j, t, s}^{\text {Load },(r)}\right]$. Also note that $\lambda_{t}^{L,(r-1)}<\lambda_{t}^{D S}$, otherwise the Load would not participate in the $r-1$ th iteration of LEM.

For $\mathrm{HV} k$, the bidding parameters are

$$
\lambda_{k, t}^{H V b i d,(r)}=\left\{\begin{array}{l}
\lambda_{t}^{H S}, \text { if } Q_{k, t}^{H V b i d,(r)} \in S_{k, t}^{H V 1,(r)} \\
\lambda_{t}^{H L,(r-1)}, \text { if } Q_{k, t}^{H V b i d,(r)} \in S_{k, t}^{H V 2,(r)}
\end{array}\right.
$$

where $S_{k, t}^{H V 1,(r)}=\left[0, \frac{1}{N_{s}} \sum_{s} Q_{k, t, s}^{H V P,(r)}-Q_{k, t}^{H V L,(r-1)}\right]$, $S_{k, t}^{H V 2,(r)}=\left[\frac{1}{N_{s}} \sum_{s} Q_{k, t, s}^{H V P,(r)}-Q_{k, t}^{H V L,(r-1)}, \frac{1}{N_{s}} \sum_{s} Q_{k, t, s}^{H V P,(r)}\right]$.

According to the iterative clearing method, which will be described in subsection IV-C, the HSSA only participates in 
the LEM after the LEM is cleared in the absence of the HSSA with $\lambda_{t}^{L n A,(r)}$ generated. Therefore, the offering/bidding parameters of the HSSA are

$$
\begin{aligned}
& \lambda_{t}^{\text {Aoffer },(r)}=\lambda_{t}^{L n A,(r)}, \text { if } P_{t}^{\text {Aoffer },(r)} \in\left[0, P_{t}^{A L S,(r)}\right] \\
& \lambda_{t}^{A b i d,(r)}=\lambda_{t}^{L n A,(r)}, \text { if } P_{t}^{A b i d,(r)} \in\left[0, P_{t}^{A L P,(r)}\right]
\end{aligned}
$$

\section{The Iterative Clearing Procedure}

The HVs are the only consumers of hydrogen in the LEM, and their trading strategies are quite easy to capture. According to (13)-(20) and (34), the HVs will purchase hydrogen from the LEM as much as they need or the LEM can supply, as long as the hydrogen price in the LEM is lower than that from the HS $\left(\mu^{H}>0\right.$ in (31)). Since the HSSA is the unique hydrogen producer in the LEM, it is capable of settling the traded amount of hydrogen in the LEM by controlling the hydrogen price in the LEM according to (31). It is quite simple to observe that the HSSA would like to set $\mu^{H}$ in (31) to a positive value close to zero. As a consequence, more attention is paid to electricity trading in the LEM here.

Fig. 3 depicts the iterative LEM clearing procedure and it consists of four steps, as follows.

Step 1. Assume the amounts of electricity accepted by all DGs and Loads are zero, the offering/bidding parameters of each DG and Load are acquired based on the optimization of utility functions. The LEM is firstly cleared in the absence of the HSSA after receiving all offering/bidding parameters from DGs and Loads, because the HSSA will use the cleared LEM price without HSSA to generate offering/bidding parameters, according to (35) and (36). The mathematical models of LEM clearing in the absence of the HSSA are as follows, and the objective is to achieve the maximum value of social welfare, represented as

$$
\begin{array}{ll}
\max _{\Gamma^{L M,(r)}} & U^{L M,(r)}=\sum_{j} \lambda_{j, t}^{\text {Loadbid },(r)} P_{j, t}^{\text {Loaddeal },(r)} \\
& -\sum_{i} \lambda_{i, t}^{\text {DGoffer },(r)} P_{i, t}^{\text {DGdeal },(r)} \\
\text { s.t. } \quad & \sum_{i} P_{i, t}^{\text {DGdeal },(r)}=\sum_{j} P_{j, t}^{\text {Loaddeal },(r)} \\
& 0<P_{i, t}^{\text {DGdeal },(r)} \leq P_{i, t}^{\text {DGoffer },(r)} \\
& 0<P_{j, t}^{\text {Loaddeal },(r)} \leq P_{j, t}^{\text {Loadbid },(r)}
\end{array}
$$

where $\Gamma^{L M,(r)}=\left\{P_{i, t}^{\text {DGdeal },(r)}, P_{j, t}^{\text {Loaddeal },(r)} ; \forall i, j, t\right\}$ is the variable set. Constraints (38)-(40) ensure that the accepted amount of electricity is in feasible regions.

Step 2. The HSSA receives cleared prices $\lambda_{t}^{\operatorname{LnA},(r)}$, optimizes strategies based on utility functions, and generates offering/bidding parameters. Then the LEM is cleared in the presence of the HSSA after receiving all offering/bidding parameters from DGs, Loads, and the HSSA. The mathematical models of LEM clearing in the presence of the HSSA are as follows, and the objective is to achieve the maximum value of social welfare, represented as

$$
\begin{aligned}
& \max _{\Gamma^{L M A,(r)}} U^{L M A,(r)}=\sum_{j} \lambda_{j, t}^{\text {Loadbid },(r)} P_{j, t}^{\text {Loaddeal },(r)} \\
& \quad+\lambda_{t}^{\text {LnA, }(r)} P_{t}^{\text {Abiddeal },(r)}-\sum_{i} \lambda_{i, t}^{\text {DGoffer },(r)} P_{i, t}^{\text {DGdeal },(r)} \\
& \quad-\lambda_{t}^{\text {LnA, }(r)} P_{t}^{\text {Aofferdeal },(r)} \\
& \text { s.t. } \quad \sum_{i} P_{i, t}^{\text {DGdeal },(r)}+P_{t}^{\text {Aofferdeal },(r)} \\
& \quad=\sum_{j} P_{j, t}^{\text {Loaddeal },(r)}+P_{t}^{\text {Abiddeal },(r)} \\
& \quad 0<P_{t}^{\text {Aofferdeal },(r)} \leq P_{t}^{\text {Aoffer },(r)} \\
& \quad 0<P_{t}^{\text {Abiddeal },(r)} \leq P_{t}^{\text {Abid },(r)} \\
& \quad(39)-(40) .
\end{aligned}
$$

where $\Gamma^{L M A,(r)}=\left\{P_{i, t}^{\text {DGdeal },(r)}, P_{j, t}^{\text {Loaddeal },(r)}, P_{t}^{\text {Abiddeal },(r)}\right.$, $\left.P_{t}^{\text {Aofferdeal },(r)} ; \forall i, j, t\right\}$ is the variable set. $P_{t}^{\text {Aofferdeal },(r)}$ and $P_{t}^{\text {Aofferdeal, }(r)}$ are the accepted offered and bid amount of electricity for the HSSA. Constraints (39)-(40) and (42)-(44) ensure that the accepted amount of electricity is in feasible regions. With the results of LEM clearing in the presence of the HSSA, the DGs, Loads, HVs, and HSSA can calculate their optimized trading strategies and utilities.

Step 3. The iteration takes place for rolling time slots through the time span, considering the situation that trading strategies can be coupled during different time slots. Two conditions affect the termination of the iterative LEM clearing process. First, all the DGs and Loads should justify the performance of adjustment in $r-1$ th iteration, as shown in Block A. If the adjustment brings no increase in utility to the DGs or Loads, the adjustment in the $r$ th iteration is to revise the adjustment in the $r-1$ th iteration, as shown in Block B. Second, if the adjustment in the $r-1$ th iteration is accepted by the DGs and Loads, they still have to decide whether to adjust the offering/bidding prices in the $r$ th iteration, as shown in Block C. If a DG finds out that the consumers' bidding amount is not entirely accepted (represented as (45)-(46)), which implies room for elevating prices, the DG will elevate the offering price in order to achieve higher utility. Similarly, if a Load finds out that the suppliers' offering amount is not entirely accepted (represented as (47)-(48)), which implies room for reducing prices, the Load will reduce the bidding price in order to achieve higher utility, as shown in Block E. Otherwise, no adjustment will be made, as shown in Block D.

$$
\begin{aligned}
& P_{i, t}^{\text {DGdeal },(r)}=P_{i, t}^{\text {DGoffer },(r)}>0 \\
& \sum_{j} P_{j, t}^{\text {Loaddeal },(r)}+P_{t}^{\text {Abiddeal },(r)} \\
& <\sum_{j} P_{j, t}^{\text {Loadbid },(r)}+P_{t}^{\text {Abid },(r)} \\
& P_{j, t}^{\text {Loaddeal },(r)}=P_{j, t}^{\text {Loadbid },(r)}>0 \\
& \sum_{i} P_{i, t}^{\text {DGdeal },(r)}+P_{t}^{\text {Aofferdeal },(r)} \\
& \quad<\sum_{i} P_{i, t}^{\text {Daffer },(r)}+P_{t}^{\text {Aoffer },(r)}
\end{aligned}
$$

Step 4. If there still needs some adjustment, go back to 


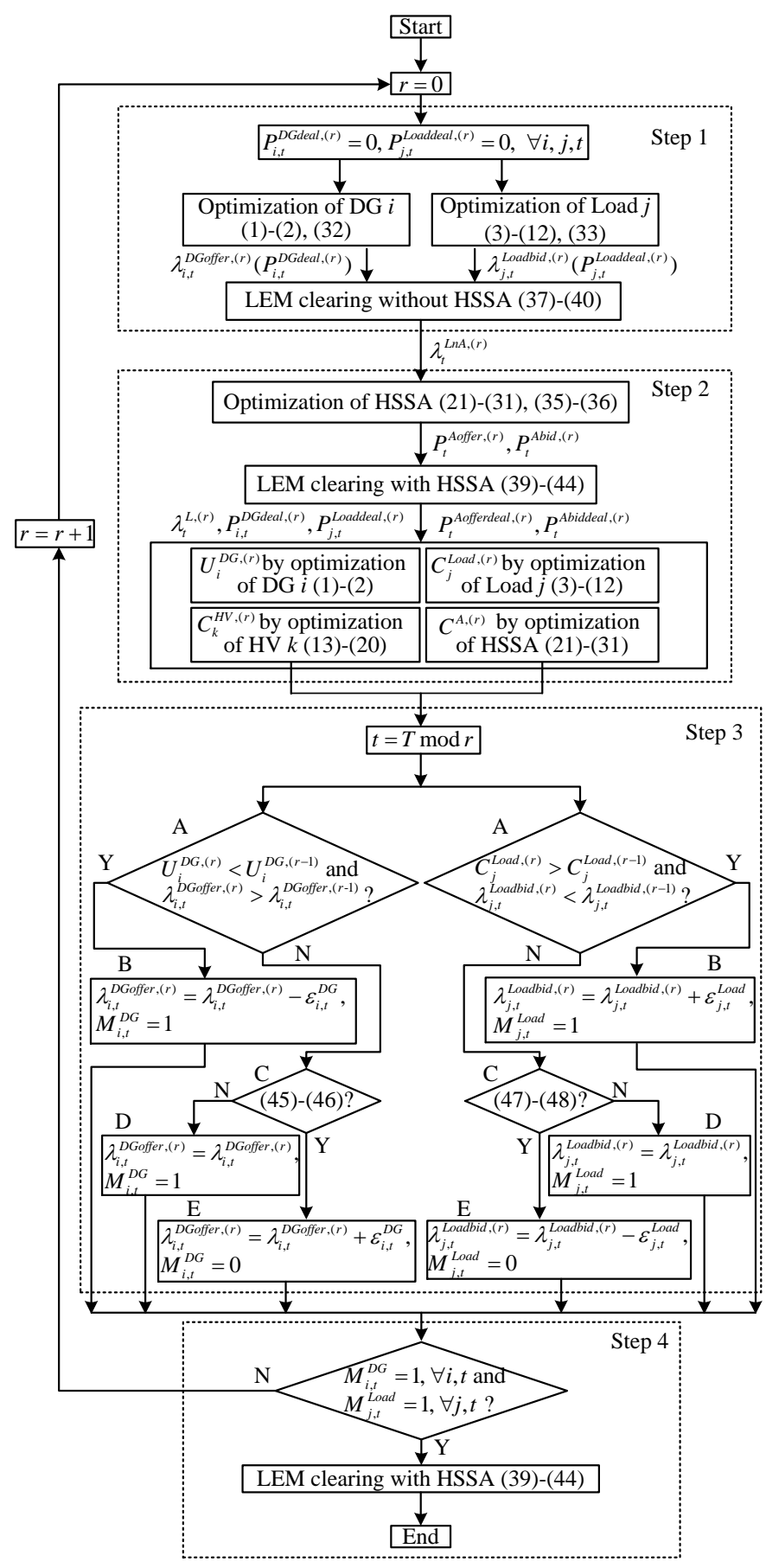

Fig. 3. The iterative LEM clearing procedure.

Step 1. Otherwise, the iteration terminates, and the LEM will be finally cleared in the presence of the HSSA with the results broadcast. Note that similar to the conventional market, when the LEM is cleared, the results will be sent to system operator for security/voltage check.

\section{Issues of Convergence}

As the unique producer of hydrogen in the LEM, the HSSA is able to determine how much hydrogen is traded in the LEM by adjusting the offering price of hydrogen, provided the offering amount does not exceed the amount HVs need.
In contrast, the electricity prices are formed through an iteration process to balance supply and demand. As stated in the Step. 3 of the algorithm, the sequences of proposed bids for each consumer $j$ generated by the algorithm are increasing, while the sequences of proposed offers for each producer $i$ are decreasing. To be specific, in a time slot when the supplier finds out its offering amount is completely accepted, whereas there is still some bidding amount of the consumers left, the supplier will elevate its offering price intending to achieve higher utility. In this case, the price will rise consequently, and then the HSSA may increase its offering amount or reduce its bidding amount. As a result, the total supply increases or the total demand decreases, until the supplier no longer observes the complete acceptance of its offering amount or an increase in its utility. It is similar for the case when the consumer observes its bidding amount is completely accepted, whereas there is still some offering amount of the suppliers left. Moreover, as stated in Section IV-A, in the case where the supply and demand curves fail to intersect, the LEM price will be settled as the price of the last unit of accepted amount of the curve that has a surplus. Therefore, the price varies during the iteration process to balance the supply and demand, and will necessarily converge. On the other hand, players have the right to choose to trade with the DisCo or in the LEM, thus prices in the LEM will not exceed those of DisCo, i.e. in the range of $\lambda_{t}^{D P}$ and $\lambda_{t}^{D S}$. This attribute introduces caps for the LEM prices.

The iterative LEM clearing procedure is a multiplayer game in which all players only care about maximizing their own utilities. In each iteration, the LEM operator clears the market to maximize the social welfare. Furthermore, players have to ensure that the adjustments introduce no decrease in their utilities. These attributes are proved as when the converged solution is achieved, no player has a unilateral incentive to deviate.

\section{CAse Study}

\section{A. Data and Approach}

In this section, the feasibility and effectiveness of the proposed iterative LEM clearing method are numerically analyzed in a 24-hr. period within a distribution network. The distribution network consists of renewable DGs with total capacity of 500kW, 100 households with $90 \mathrm{EVs}$ and $90 \mathrm{HVs}$, and an HSSA.

Data regarding renewable DG generation are acquired from [2]. Note that there is no power generation before time slot 3 or after time slot 19. Data of three sorts of tasks of Loads are obtained from [35]-[36], with an assumption that the starting/ending time requirements of different tasks are in Gaussian distribution with a standard deviation of 1-3 hrs. For the detailed data of Loads, see Appendix. Driving patterns for EVs and HVs are randomly generated based on statistical data from the National Household Travel Survey (NHTS) data set in the U.S. [37]. All HVs are assumed to be Hyundai ix 35 FUEL CELL vehicles, detailed parameters of which are illustrated in [38]. The HSSA is assumed to have 20 HSSs, the parameters of which are illustrated in [29]. To consider the uncertainties 


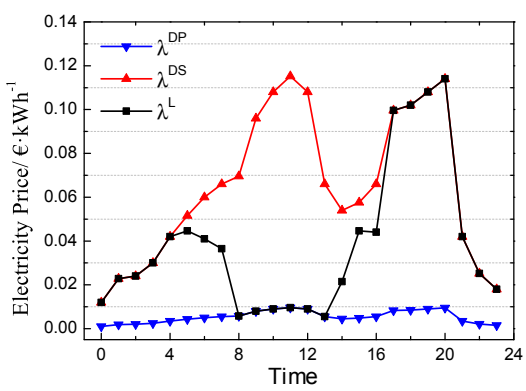

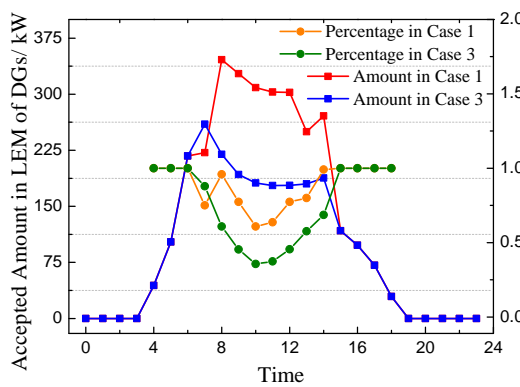

Fig. 4. Electricity price of the LEM for Case 1.Fig. 5. DG generation in the LEM.

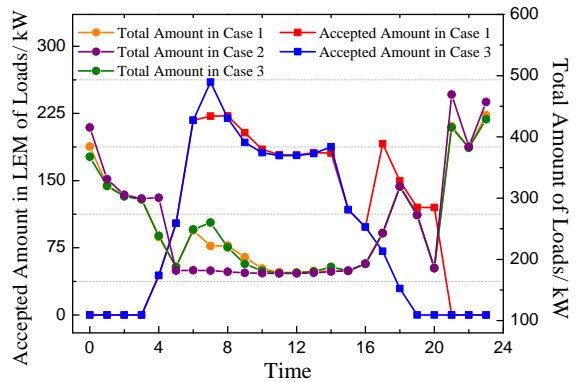

Fig. 6. Load consumption in LEM.

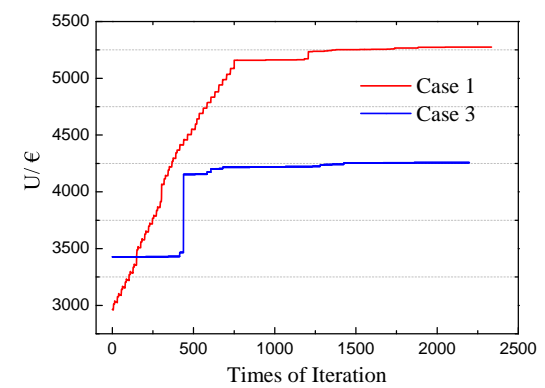

Fig. 7. Convergence of the method.

of renewable DG generation, demand of Loads and HVs, we generate 1000 scenarios and reduce them to 5 [39]. Prices for the DisCo are acquired from [9]. Hydrogen price from the HS is set to $1.35 € / \mathrm{kg}$. The adjustment of offering/bidding prices for DGs $\left(\varepsilon_{i, t}^{D G}\right)$ and Loads $\left(\varepsilon_{j, t}^{\text {Load }}\right)$ are all set to $0.001 € / \mathrm{kWh}$.

To clearly illustrate the applicability and efficiency of the proposed LEM, three cases are compared.

Case 1: Adopt LEM and HSSA exists in the LEM.

Case 2: Not adopt LEM and players only trade with $\mathrm{DisCo} / \mathrm{Hs}$.

Case 3: Adopt LEM but no HSSA exists in the LEM.

Note that to apply the mathematical models in Section III to Case 2, just set the quantity offered/bid in the LEM by each player as 0 .

\section{B. Local Energy Market Clearing}

The market prices for Case 1 are shown in Fig. 4. The LEM prices stay in the range of the purchasing and selling electricity prices of the DisCo. For time plots when the LEM price equals the purchasing price of the DisCo, bids in the LEM are completely accepted, whereas offers are not. For time plots when the LEM price equals the selling price of the DisCo, offers in the LEM are completely accepted, whereas bids are not. For time plots when the LEM price lies between the purchasing and selling prices of the DisCo, neither offers nor bids are completely accepted.

Case 1 is further compared to the other two cases. Fig. 5 compares the DG generation of Cases 1 and 3. It is observed that the LEM in Case 1 adopts $33.39 \%$ more DG generation than in Case 3. Therefore, the LEM combining the HSS promotes the local integration of renewable energy. Fig. 6 further demonstrates the load consumption of the three cases. About $20.14 \%$ more demands are met in the LEM in Case 1 than in Case 3. Furthermore, the peak demand is $7.21 \%$ and

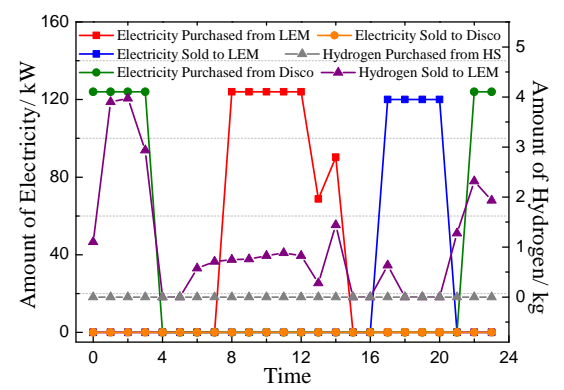

Fig. 8. Electricity and hydrogen production/consumption of the HSSA.

8.60\% lower in Cases 1 and 3 than in Case 2, respectively. Therefore, the LEM helps to reduce the peak value of demand.

Fig. 7 compares the overall utility in Cases 1 and 3. The DGs and Loads are considered as two sorts of utility-seeking players and they attempt to agree on a settlement point, which is the market clearing point. According to Nash bargaining theory, the settlement point is where the product of increases in utilities of the DGs and Loads reaches the maximum value, represented as [40]

$$
\begin{array}{r}
U=\left(\sum_{i} U_{i}^{D G,[\text { withLEM }]}-\sum_{i} U_{i}^{D G,[\text { without LEM }]}\right) \\
\cdot\left(\sum_{j} C_{j}^{\text {Load },[\text { without } L E M]}-\sum_{j} C_{j}^{\text {Load },[\text { withLEM }]}\right)
\end{array}
$$

As shown in Fig. 7, the value of overall utility $U$ varies during the iteration process and converges to the maximum value. In case 1, with the help of the HSSA, the overall utility is improved by $77.96 \%$. The LEM is thus proved to be able to improve the players' utilities.

The strategies of the HSSA are demonstrated in Fig. 8. The HSSA never sells electricity to the DisCo because the electricity purchasing prices of the DisCo are always no higher than those in the LEM. The HSSA never purchases hydrogen from the HS because the cost of purchasing hydrogen from the HS is higher than that of producing hydrogen with electricity, which is acquired when electricity prices are relatively low.

\section{Sensitivity Analysis}

To evaluate the influences of different DG and Load capacities on the LEM clearing, the capacities of the DGs and Loads are set to 0.50, 0.75, 1.25, 1.50 and 1.75 times those in Case 1, respectively. The results are shown in Figs. 9 and 10. The percentage of DG absorption refers to the ratio of accepted DG amount in the LEM to the total DG generation. The equivalent 


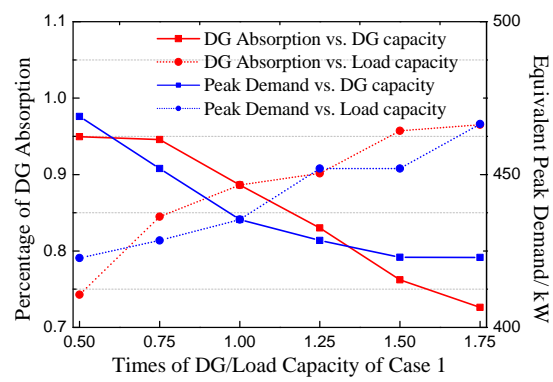

Fig. 9. DG absorption, equivalent peak demand vs. DG and Load capacities.

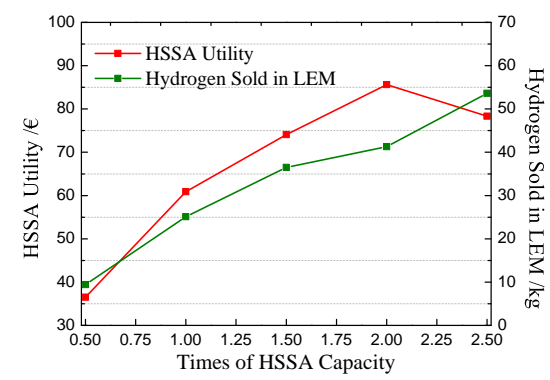

Fig. 12. HSSA utility and hydrogen sold in LEM vs. HSSA capacity.

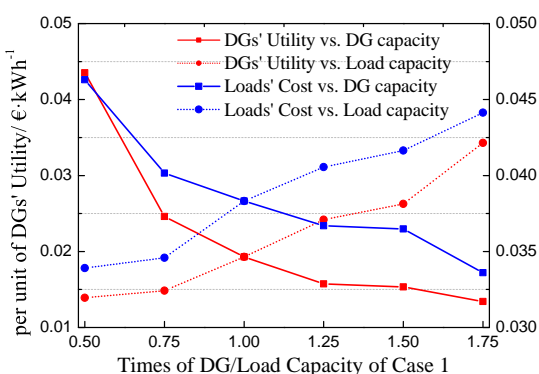

Fig. 10. DG/Loads utility vs. DG and Load capacities.

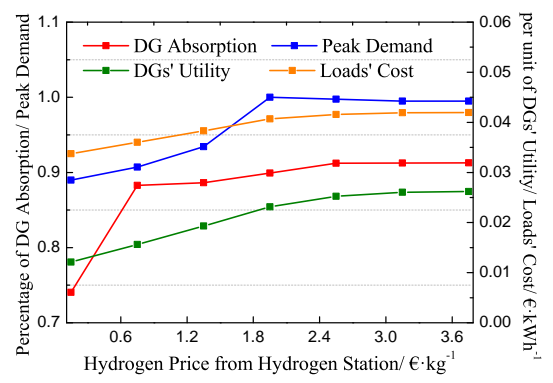

Fig. 13. DG absorption, peak demand, DGs'/ Load utility vs. Hydrogen price from HS.

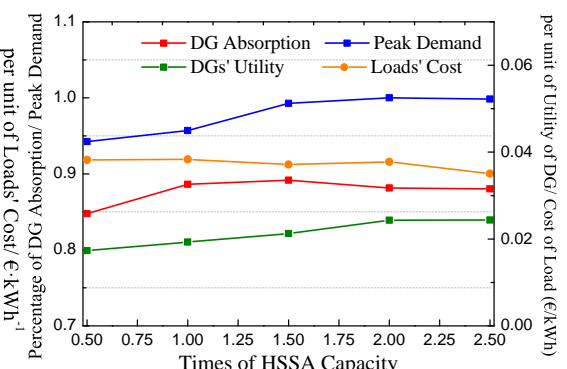

Fig. 11. DG absorption, peak demand, DGs'/Loads utility vs. HSSA capacity.

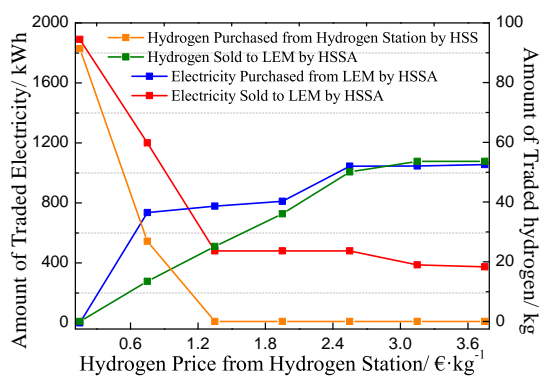

Fig. 14. Amount of traded electricity and hydrogen vs. Hydrogen price from HS. peak demand refers to the peak demand when Load capacity is converted to the value in Case 1 so as to remove the effects of differences in Load capacity. As shown in Figs. 9 and 10, the increase in a DG's capacity leads to a decrease in DG absorption, peak demand, the DGs' utility and the Loads' cost, whereas the increase in a Load's capacity results in an increase in DG absorption, peak demand, DGs' utility and Loads' cost. This is because the increase in a DG's capacity results in lower LEM prices, whereas the increase in a Load's capacity results in higher LEM prices. This demonstrates that both DGs and Loads are not willing to enlarge their trading capacities in the LEM with consideration of their utilities. The LEM operator should balance the increase in capacities of both producers and consumers to make the LEM economically attractive so as to enhance the market size. For example, to ensure a certain level of DG absorption, the timing of introducing DG capacity is extremely critical in order to reach the optimal inter-temporal social welfare. To be specific, expansion of the DG's capacity should happen in response to an increase in demand. Otherwise, the DGs would choose to trade with the DisCo and thus the decentralized nature of the system is weakened.

The HSSA's capacity is also adjusted to evaluate its impact on DG absorption, peak demand, DGs' utility and Loads' cost, as shown in Fig. 11. The peak demand here is calculated as the ratio of equivalent peak demand to the maximum value of equivalent peak demand. The DG absorption and peak demand reach the highest value when HSSA capacity is 1.50 and 2.00 times that in Case 1, respectively. The DGs' utility increases and the Loads' cost decreases slightly along with increasing HSSA capacity. Fig. 12 further demonstrates the HSSA's utility and the amount of hydrogen sold in LEM. With the increasing HSSA capacity, the HSSA's utility reaches the maximum value when the HSSA capacity is 2.00 times that in Case 1, while the amount of hydrogen sold in the LEM gradually increases. This demonstrates that the HSSA can set its capacity at the optimal value to achieve the highest utility.

The hydrogen price from the HS is a key factor for generating offering/bidding parameters of the HSSA and HVs. As shown in Fig. 13, the increasing hydrogen price results in increasing DG absorption, DGs' utility and Loads' cost, whereas peak demand increases at first and remains steady afterwards. This is because the HSSA will purchase more electricity from the LEM and less hydrogen from the HS with the increasing hydrogen price, as shown in Fig. 14.

\section{Computational Issues}

With regard to computational issues, all problems are implemented on $\mathrm{C}++$ and the related MILP problems are solved by CPLEX, on a computer with an Intel Core i7 $3.60 \mathrm{GHz}$ $\mathrm{CPU}$ and $8 \mathrm{~GB}$ of RAM. The averaged iteration time of the problems is 2273 , and the averaged running time is $825 \mathrm{~s}$.

\section{CONCLUSION}

To provide an efficient market mechanism for integrating DERs in a distribution-level network, this paper establishes an LEM framework in which electricity and hydrogen are traded. Players including renewable DGs, Loads, HVs, and an HSS participate in the LEM by submitting offers/bids based on their own preferences and profiles according to the utility functions. An iterative LEM clearing method is proposed based on the merit order principle, and it not only avoids complex calculation induced by a centralized decision process but also preserves players' privacy. Case studies are conducted to justify the applicability and efficiency of the LEM. The results show that the LEM promotes the local integration of renewable energy by $33.39 \%$, reduces peak demand by $7.21 \%$, 
and improves players' utilities by $77.96 \%$. Sensitivity analysis is also implemented to analyze the influences on LEM clearing results of the capacities of DGs, Loads, and the HSSA, as well as price of hydrogen from the HS.

\section{APPENDIX}

The data regarding three sorts of tasks of Loads are illustrated in Tables I, II and III.

TABLE I: Data on non-interruptible and non-deferrable tasks

\begin{tabular}{l|ll}
\hline \hline Task & Power $(\mathrm{kW})$ & Total usage period $(\mathrm{h})$ \\
\hline Refregerator & 1.666 & 24 \\
Telephone & 0.005 & 24 \\
Others & 0.05 & 24 \\
\hline \hline
\end{tabular}

TABLE II: Data on interruptible and deferrable tasks

\begin{tabular}{l|lllll|}
\hline \hline Task & $\begin{array}{l}\text { Power } \\
(\mathrm{kW})\end{array}$ & $\begin{array}{l}\text { Total } \\
\text { usage } \\
\text { period } \\
(\mathrm{h})\end{array}$ & $\begin{array}{l}\text { Averaged Averaged Standard } \\
t_{j, n_{j}}^{i d 1}\end{array}$ & $t_{j, n_{j}}^{i d 2}$ & $\begin{array}{l}\text { deviation of } \\
t_{j, n_{j}}^{i d 1} / t_{j, n_{j}}^{i d 2} \\
(\mathrm{~h})\end{array}$ \\
\hline $\mathrm{AC}$ & 1.2 & 7.25 & $16: 45$ & $08: 30$ & 3 \\
Oven & 1.2 & 0.5 & $19: 00$ & $20: 45$ & 1 \\
Microwave & 0.2 & 0.25 & $18: 45$ & $20: 00$ & 1.5 \\
Iron & 1.2 & 0.5 & $19: 00$ & $19: 45$ & 1 \\
Toaster & 0.2 & 0.25 & $07: 15$ & $08: 30$ & 2 \\
Kettle & 1 & 0.5 & $06: 45$ & $07: 45$ & 2.5 \\
Dishwasher & 1.32 & 0.5 & $20: 00$ & $22: 45$ & 3 \\
Hairdryer & 0.9 & 0.25 & $20: 30$ & $21: 30$ & 2.5 \\
Others & 0.12 & 0.5 & $17: 00$ & $9: 45$ & 3 \\
\hline \hline
\end{tabular}

TABLE III: Data on non-interruptible and deferrable tasks

\begin{tabular}{|c|c|c|c|c|c|}
\hline Task & $\begin{array}{l}\text { Power } \\
(\mathrm{kW})\end{array}$ & $\begin{array}{l}\text { Total } \\
\text { usage } \\
\text { period } \\
\text { (h) }\end{array}$ & $\begin{array}{l}\text { Averaged } \\
t_{j, n_{j}}^{n i d 1}\end{array}$ & $\begin{array}{l}\text { Averaged } \\
t_{j, n_{j}}^{n i d 2}\end{array}$ & $\begin{array}{l}\text { Standard } \\
\text { deviation } \\
\text { of } t_{j, n_{j}}^{n i d 1} \\
\text { (h) }\end{array}$ \\
\hline TV & 0.083 & 6.75 & $16: 00$ & $23: 30$ & 3 \\
\hline $\begin{array}{l}\text { Washing } \\
\text { machine }\end{array}$ & 1.4 & 1 & $17: 45$ & $21: 30$ & 1.5 \\
\hline Computer & 0.15 & 2.25 & $19: 30$ & 23:00 & 2 \\
\hline Others & 0.09 & 3 & $15: 30$ & 09:30 & 3 \\
\hline
\end{tabular}

\section{REFERENCES}

[1] Z. Ding, Y. Guo, D. Wu, and Y. Fang, "A market based scheme to integrate distributed wind energy," IEEE Trans. Smart Grid, vol. 4, pp. 976-984, Jun. 2013.

[2] D. Nguyen, and L. Le, "Optimal bidding strategy for microgrids considering renewable energy and building thermal dynamics," IEEE Trans. Smart Grid, vol. 5, pp. 1608-1620, Jul. 2014.

[3] Q. Wang, et al, "Review of real-time electricity markets for integrating distributed energy resources and demand response," Appl. Energy, vol. 138, pp. 659-706, Jan. 2015.

[4] M. Bayat, K. Sheshyekani, M. Hamzeh, and A. Rezazadeh, "Coordination of distributed energy resources and demand response for voltage and frequency support of MV microgrids," IEEE Trans. Power Syst., vol. 31, pp. 1506-1516, Mar. 2016.
[5] I. Perez-Arriaga, "The transmission of the future: the impact of distributed energy resources on the network," IEEE Power and Energy Mag., vol. 14, pp. 41-53, Jul/Aug. 2016.

[6] J. Mohammadi, A. Rahimi-Kian, and M. Ghazizadeh, "Aggregated wind power and flexible load offering strategy," IET Renew. Power Gener, vol. 5, pp. 439-447, Nov. 2011.

[7] J. Lopez-Lezama, A. Padilha-Feltrin, J. Contreras, and J. Munoz, "Optimal contract pricing of distributed generation in distribution networks," IEEE Trans. Power Syst., vol. 26, pp. 128-136, Feb, 2011.

[8] A. Safdarian, et al, "Optimal electricity procurement in smart grids with autonomous distributed energy resources," IEEE Trans. Smart Grid, vol. 6, pp. 2975-2984, Nov. 2015.

[9] C. Zhang, et al, "Trading strategies for distribution company with stochastic distributed energy sources," Appl. Energy, vol. 177, pp. 625635, Sep. 2016.

[10] G. Hug, S. Kar, and C. Wu, "Consensus + innovations approach for distributed multiagent coordination in a microgrid," IEEE Trans. Smart Grid, vol. 6, pp. 1893-1903, Jul. 2015.

[11] D. Papadaskalopoulos, and G. Strbac, "Decentralized participation of flexible demand in electricity market—Part 1: market mechanism," IEEE Trans. Power Syst., vol. 28, pp. 3658-3666, Nov, 2013.

[12] Z. Li, Q. Guo, H. Sun, and H. Su, "ADMM-based decentralized demand response method in electric vehicle virtual power plant," in IEEE PES General Meeting, 2016, pp. 1-5.

$\mp 13]$ K. Kok, and S. Widergren, "A society of devices: integrating intelligent distributed resources with transactive energy," IEEE Power and Energy Mag., vol. 14, pp. 34-45, May/Jun. 2016.

[14] Y. Parag, and B. Sovacool, "Electricity market design for the prosumer era," Nature Energy, vol. 1, pp. 1-6, Mar. 2016.

[15] P. Silva, et al, "The impact of smart grid prosumer grouping on forecasting accuracy and its benefits for local electricity market trading," IEEE Trans. Smart Grid, vol. 5, pp. 402-410, Jan. 2014.

[16] S. Sikdar, and K. Rudie, "A decentralized electricity market model: an electric vehicle charging example," in IEEE Canadian Conf. Elec. and Comp. Engi., 2014, pp. 1-6.

[17] P. Shamsi, H. Xie, A. Longe, and J. Joo, "Economic dispatch for an agent-based community microgrid," IEEE Trans. Smart Grid, vol. 7, pp. 2317-2324, Sep. 2016.

[18] D. Wiyono, et al, "Novel energy exchange models and a trading agent for community energy market," in Int. Conf. EEM, 2016, pp. 1-5.

[19] L. Bird and B. Swezey, "Green power marketing in the United States: A status repoty," National Renewable Energy Laboratory, 2009.

[20] Powerpeers, Energie van elkaar [Online]. Available: https://www.powerpeers.nl/.

[21] S. Nykamp, M. Bosman, A. Molderink, J. Hurink and G. Smit, "Value of storage in distribution grids-competition or cooperation of stakeholders?" IEEE Trans. Smart Grid, vol. 4, pp. 1361-1370, Sep. 2013.

[22] H. Ding, P. Pinson, Z. Hu, and Y. Song, "Optimal offering and operating strategies for wind-storage systems with linear decision rules," IEEE Trans. Power Syst., vol. 31, pp. 4755-4764, Nov. 2016.

[23] T. AlSkaif, et al, "Reputation-based joint scheduling of households appliances and storage in a microgrid with a shared battery," Energy and Building, vol. 138, pp. 228-239, Mar. 2017

[24] H. Akhavan-Hejazi, et al, "Optimal operation of independent storage systems in energy and reserve markets with high wind penetration," IEEE Trans. Smart Grid, vol. 5, pp. 1088-1097, Mar. 2014.

[25] S. Shafiee, et al, "Risk-constrained bidding and offering strategy for a merchant compressed air energy storage plant," IEEE Trans. Power Syst., 2016, in press.

[26] L. Valverde, F. Rosa, C. Bordons, and J. Guerra, "Energy management strategies in hydrogen smart-grids: a laboratory experience," Int. J. of Hydr. Energy, vol. 41, pp. 13715-13725, Aug. 2016.

[27] M. Korpas, and T. Holen, "Operation planning of hydrogen storage connected to wind power operating in a power market," IEEE Trans. Energy Convers, vol. 21, pp. 742-749, Sep. 2006.

[28] C. Brunetto, and G. Tina, "Optimal hydrogen storage sizing for wind power plants in day ahead electricity market," IET Renew. Power Gener. vol. 1, pp. 220-226, Dec. 2007.

[29] S. Nojavan, K. Zare, and B. Mohammadi-Ivatloo, "Selling price determination by electricity retailer in the smart grid under demand side management in the presence of the electrolyser and fuel cell as hydrogen storage system," Int. J. of Hydr. Energy, 2016, in press.

[30] S. You, J. Hu, Y. Zong, and J. Lin, "Value assessment of hydrogenbased electrical energy storage in view of electricity spot market," J. of Mod. Power Syst. and Clean Energy, vol. 4, pp. 626-635, Oct. 2016. 
[31] S. Kazempour, A. Conejo, and C. Ruiz, "Strategic generation investment using a complementarity approach," IEEE Trans. Power Syst., vol. 26, pp. 940-948, May. 2011.

[32] Q. Wang, C. Zhang, J. Wang, P. Pinson, and J. Oestergaard "Real-time trading strategies of proactive DISCO with heterogeneous DG owners," IEEE Trans. Smart Grid, 2016, in press.

[33] N. Paterakis, O. Erdinc, I. Pappi, A. Bakirtzis, and J. Catalao, "Coordinated operation of a neighborhood of smart households comprising electric vehicles, energy storage and distributed generation," IEEE Trans. Smart Grid, vol. 7, pp. 2736-2747, Nov. 2016.

[34] D. Kirschen, and G. Strbac, Fundamentals of power system economics, 1st ed. John Wiley \& Sons, 2004.

[35] J. Wang, M. Shahidehpour, and Z. Li, "Security-constrained unit commitment with volatile wind power generation," IEEE Trans. Power Syst., vol. 23, pp. 1329-1327, Aug. 2008.

[36] O. Erdinc, "Economic impacts of small-scale own generating and storage units, and electric vehicles under different demand response strategies for smart households," Appl. Energy, vol. 126, pp. 142-150, Aug. 2014.

[37] U.S. Department of Transportation, 2009, National Household Travel Survey [Online]. Available: http://nhts.ornl.gov/download.shtml.

[38] Ix35 Fuel Cell, Hyundai [Online]. Available: http://worldwide.hyundai.com/WW/Showroom/Eco/ix35-FuelCell/PIP/index.html\#wrap.

[39] H. Heitsch, et al, "Scenario reduction algorithms in stochastic programming," Comp. Opti. and Appl., vol. 24, pp. 187-206, Feb. 2003.

[40] N. Yu, L. Tesfatsion, and C. Liu, "Financial bilateral contract negotiation in wholesale electricity markets using Nash Bargaining Theory," IEEE Trans. Power Syst., vol. 27, pp. 251-267, Feb. 2012. 